\title{
Space Portrait: Establishment of a Lifestyle-Directed Visualized Model for Interior Design
}

\author{
Chiung-Hui Chen*1 \\ ${ }^{1}$ Assistant Professor, Department of Visual Communication Design, Asia University, Taiwan, R.O.C.
}

\begin{abstract}
Effective interior design service relies on users' needs and the agreements between designers and users; this requires good communication between the two parties. Therefore, establishing an interactive model to record user involvement in the design process, as well as visually analyzing the user's lifestyle and needs, warrants exploration. This study targeted specific interior design cases and the daily lives of users. The research introduces a sphere activity change map that uses information visualization to understand users' daily activities. This model offers a life-model-based information tree as a tool to highlight the space spheres individual family members emphasize. The tool then visualizes the distribution of the average time family members spend in each space sphere.
\end{abstract}

Keywords: interior design; lifestyle patterns; information visualization; aesthetic computing

\section{Introduction}

\subsection{Background of the Study}

Architecture design refers to any design activity specific to the purposes of architecture, including activities intended to meet requirements for environment, usage, and visualization. Such activity concerns historical, cultural, and symbolic meanings specific to architecture. The scope of architecture design includes appearance design and interior design, and it can be further extended to the relationship between architecture and the environment. Interior design is, then, the re-creation of the interior environment of architecture. In the field of residential space design, the styles and preferences of inhabitants are transformed into design concepts and employed in the process of design, thus converting interior spaces to reflect life values. This activity emphasizes the tastes of families and individuals, expresses the user's attitude toward life, and provides a more cozy living space. In terms of functionality, therefore, architectural design provides an existing space, but lifestyle and interior space have to be refined through detailed design. In other words, interior design not only beautifies architectural interfaces, but also re-creates interior functionality and space form.

Regarding interior design service, in the traditional process, a designer uses his or her knowledge and

*Contact Author: Chiung-Hui Chen, Assistant Professor, Department of Visual Communication Design, Asia University, 500 Lioufeng Rd., Wufeng, Taichung 41354, Taiwan, R.O.C. Tel: +886-4-23323456-1072 Fax: +886-4-23316699

E-mail: 7451616@gmail.com

(Received April 1, 2014 ; accepted October 15, 2014) skills to convert to concrete form the concept generated from the ideas and needs of the user (client). The final product is created through a very time-consuming process of repeated communication and modification. Although this process has been accelerated by computer graphics software, repeated discussions and confirmations with users are still required. Innovative interior design service must consider user needs and reach consensus with the user. Users should be given sufficient design-related information, thus allowing them to understand the style elements of their needs and participate in the provision of innovative design service. The time and cost of communication can therefore be reduced, and the user may gain a sense of achievement.

\subsection{Objectives of the Study}

Building a platform that can document the user's participation in design communication, as well as visualize his or her life model and design requirements, warrants further study and development. To produce a design object that reflects the user's experience and meets his or her expectations, designers need more messages that influence the interaction between the user and the design object. Combining these messages with the designer's expertise helps him or her not only explore the user's experience and needs, which are introduced into the design, but also investigate innovative design concepts early in the process. To save the time spent on searching for design data and converting the data acquired from the user's life model, data are visualized into design information with specific meanings. Once the designer understands the visualized information, it is converted to design knowledge and eventually applied in the actual design. In other words, a space user's life model is analyzed 
using visualization techniques to create a system modeled after interior design knowledge. The user documents his or her personal experience in a model requirement chart, allowing the researcher to analyze interrelations between analysis documents and identify the logic and substance of the data conversion. The documented data are then transformed into design information for sharing and reuse. A professional interior designer can sort out the correlations among user preferences, life patterns, and design specifications, thus determining the critical elements in the design process.

\section{Related Studies and Model Construction}

\subsection{Technology Used in Information Visualization}

Information visualization is a unique academic discipline. It combines multiple disciplines, including computer science, psychology, semiotics, graphics design, interaction design, and art. It pays close attention to creating effective interaction between abstract data and users in a visual and metaphorical way. Abstract data is characterized by a lack of space initiative; as such, the visualized presentation of abstract data and the development of new visual metaphors and data presentation methods are challenging for information visualization researchers. Card et al. (1999) define information visualization as the visible presentation of the interaction between abstract data for the purpose of better understanding. As such, visualization can improve the interaction between users and abstract data as well as users' degrees of awareness. On the other hand, information visualization consists of two concepts - data and visualization. This means that objects, concepts, or abstract data are expressed as images instead of text. Because of the multiple types and various structures of data, Shneiderman (1992) divides data into seven basic types: one-dimensional, two-dimensional, three-dimensional, multi-dimensional, time, tree, and network.

Visualization can be further expressed as visually presenting abstract data using human feeling and cognition. There have been several relevant case studies on information visualization technology regarding efficacy and the type of visualization. Treemapping is the type of visualization technology proposed by Shneiderman (1992). This method uses skillful spatial allocation techniques to present hierarchical data structures in nested rectangles. The size of the rectangle space expresses the relationships among certain attributes of the information object. The sunburst type proposed by Stasko et al. (2000) is similar to a visualization technique called space filling, and is used to present the information in file directories. The circular image in the center represents the root directory or the top level of hierarchical information. This circle spreads outward from the center according to the level or depth of the directory, while the divisions of the circles correspond to the sizes of the directories or documents. Hyperbolic viewer is a visualization technique proposed by Lamping et al. (1995) and is used to visualize large hierarchies. In this technique, data are presented in a tree structure and placed on a hyperbolic plane. Through interaction with the user, important information is moved to the center of the screen and magnified into a fisheye view. The relationships between objects in the hierarchy are thus clearly observed.

Skog et al. (2003) developed the concept of blending information into environmental design. The information art expressed in weather forecasts thus resembles a piece by Mondrian. Each rectangle represents one day, and the size of the rectangle represents the temperature of the day. The color represents weather conditions with yellow for sunny, blue for rainy, and red for cloudy. The date is calculated and displayed left to right, from top to bottom, in the sequence of tomorrow, the day after tomorrow, and so forth. By communicating information in a public space using an information display object, this design becomes more than just a pure display object. The "7000 oaks and counting" project developed by Holmes (2007) displays energy consumption through information visualization. The values of various energy flows provided by a building's central control system are acquired through customized software. These values are saved in a server and converted to data showing the carbon footprint created by human activity in real time. Electricity consumption is displayed metaphorically through the idea that trees can absorb carbon dioxide. The more electricity is consumed, the more trees are to be planted. Such information is presented as a display in a hallway. This artwork was intended to explore methods to help people see and understand the underlying information hidden in a building. Based on the analysis and comparison of visual information and the interviews with residents, energy consumption information obtained from visualization can indeed help people understand electricity consumption and save energy, thus promoting sustainable development. Color design is the best strategy for creating an attractive product economically and effectively, especially in the field of interior design. Huang et al. (2006, 2009) developed a color-plan consulting and visual-simulating system to help designers produce optimum colors for components and decorative patterns. A color plan can help designers find good color schemes, and color combinations can have a strong influence on how clients feel.

\subsection{Definition of the Life Model}

Humans develop close interactions with their environments, which involve standing behaviors and the locations where such behaviors occur. Environmental behaviors are thus multifaceted (Lewin, 1943). Barker (1968) proposes four factors in "Behavior Settings": the user, the standing pattern 
of behavior, the physical environment, and a specific period of time. Lang (1987) also proposes that behavior settings are standing patterns of behavior that appear repeatedly in a specific place and time. If different users access the place, the behavior settings do not disappear as a result. They will only disappear when the physical environment changes. In other words, the standing pattern of behavior has a close relationship with time. Further exploring the perspective of time, Wicker (1987) proposes a dynamic change model for behavior settings. He describes behavior settings as segmented in a time sequence with composition factors as the major points of entry. From the initial gathering to the standing pattern of behavior to the ending behavior, this is a process of dynamic change in behavior settings.

As such, the time during which behavior settings appear may be short or lengthy. For the purposes of analysis, the records obtained during the study must be well controlled during the investigation. Wicker (1992) and Schoggen (1989) also point out that to describe confirmed behavior settings objectively, they must be divided into four parts to discuss the relationships between the parts and all information must be recorded. These four parts include the user's frequent behaviors and classifications, the amounts and attributes of behavior settings, the time and duration of occurrences, and the location and size of the dimensions. The life model in this study is thus defined as a cycle consisting of the generation, growth, change, fading, and ending of a user's behavior in a residential environment within a time sequence. In studying the value of the life model, Højrup (2003) stresses that designers should not only focus on understanding the user's life model but also integrate the user's preferences, family, and future demands with the characteristic information of a house, thus creating a complete map of the user's life model. To develop a joint design service based on a life model, users must share their ideas and opinions, especially in the initial design phase. Communication and reflection are crucial to the core of the design. In summary, this study employs the theory of behavior settings as the infrastructure for investigation, thus enabling space users to record behaviors that occur at home and the locations where such behaviors occur during the test period. The users' life models for a single day and over a period of time are analyzed accordingly.

\subsection{Relationship between the Life Model and Information Visualization}

A vast amount of information has been involved in the development of human cognitive abilities. Accordingly, Card et al. (1999) created the term information visualization, which involves properly organizing mass data to gain insight, find answers, discover correlations among data, and understand things otherwise difficult to perceive. A few studies (Spence, 2001; Bederson and Shneiderman, 2003; Colin, 2004) have confirmed that information visualization improves a cognition system's understanding by visually presenting abstract data. These studies also indicate that a perception system achieves understanding much faster than a cognition system, with visual capacity providing understanding in the shortest amount of time. Exploring the visualization of data allows people to participate in the process of data mining. As a result, relevant subjects of study emerge, such as architecture data mining, architecture design knowledge, and knowledge mining. In design analysis and decision making (Cawthon and Vande, 2007), design data visualization can assist architecture design data mining and knowledge mining through data classification and capture, using methods such as cross-examination and statistical analysis.

In his book The Ten Books on Architecture (De Architectura), the ancient Roman architect Vitruvius (1960) identifies three architectural design principles that should adhere to the following: completeness, practicality, and aesthetics. Vande Moere and Purchase (2011) suggest that these principles can be applied in information design and various applications that are suitable for information design. They also indicate that good visualized content should be very complete. In other words, the form of the design must match the information described by the design. Moreover, visualized content should be practical so that the user can derive meaning from it. Finally, similar to any other design, information design should be aesthetically appealing, drawing the user's attention and providing an overall pleasant visual experience. The three design principles proposed by Vande Moere and Purchase (2011) provide a solid foundation for judging the value of information visualization. This study is founded upon the above-mentioned viewpoints, as well as Colin's (2004) four steps of visualization: collecting and storing data, preprocessing and converting data into an intelligible form, displaying images generated by the graphical engine on a screen, and human observation and cognition of the image. Feedback also exists between these steps, whose correlations are shown in Fig.1. Quantified data are used to express certain characteristics with symbols in lieu of the digital expression of data. In terms of visual meanings and perception, an interior designer is more inclined to accept symbols than text, mainly because he or she can quickly understand the data and grasp their characteristics with the help of symbols. As for the data itself, the area scale, color, length, and quantity of the image are used to express content. The created image symbols replace textual explanations through visual metaphors. The project interior designer can thus review the digital data, provide opinions, and offer higher-quality design service.

\section{Methodology: Exploring the Life Model 3.1 Client Background}

This study uses a nuclear family as a case study. 


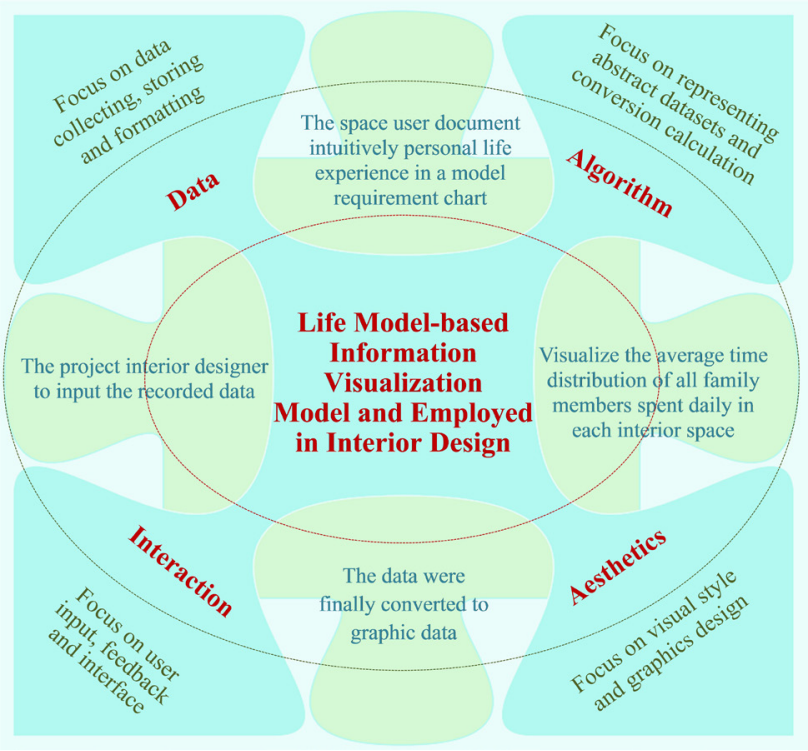

Fig.1. Life-Model-Based Information Visualization Model

There are three members in the family: Mr. Peng, Mrs. Peng, and their son, who is a senior in high school. Mr. Peng, 47, owns a medium-sized company. His hobbies include reading and travel. His friends often visit him at home. He likes to chat over coffee, and he spends much of his time before a computer. He occasionally plays basketball. Mrs. Peng, 45, stays home most of the time. She is a very good cook. She sometimes invites friends over for a meal. She likes coffee, arts, and crafts. She often goes for walks in the country and travels overseas. The Peng family home is located on Main Street; it has two floors and is 1,800 square feet. The living room, dining room, kitchen, and rest room are on the first floor, while the master bedroom, son's room, study room, bathroom, and balcony are on the second floor.

\subsection{Space Encoding}

Different cultures account for different space forms under different environments. Any space has a sphere depth, and the defined sphere is the result of the encompassed boundary. This study defines space sphere depth according to Hillier and Hanson's (1984) and Hillier's (1996) theories of sphere structure. Hillier (1996) suggests that sphere depth in space is obtained by observing the physical environment, the walking distance in space, and the accessibility of visual permeability. He also indicates that space relations are not geometrical but topological. The study of space structure is thus rid of plane-based study, and space encoding is rendered and numeralized into symbols and a mathematical system with a single meaning for better exploration of the correlation between spaces. Thus, when a space has lower accessibility and the behavior is more private, the sphere depth is deeper. Conversely, when more people share a space and the space has higher accessibility, the sphere depth is shallower.
This study is guided by the degree of privacy in a behavior and divides the sphere structures of living spaces in the case study into four levels (i.e., space coding) on a scale ranging from "private" to "open." Living spaces therefore include several independent space units: rest room, master bedroom, child's bedroom, kitchen, study, dining room, living room, balcony, and yard. Each room is described as below. The bathroom and bedrooms are space encoded as No. 1 because they belong to the same category, having the highest levels of privacy. The kitchen and study are spaces where independent behaviors occur, but they may be shared by family members, thus leading to cooperative behavior. They are space encoded as No. 2 . The dining and living rooms are spaces where family members and visitors gather for cooperative behaviors; they are space encoded as No. 3. The balcony and yard can be seen by pedestrians; they are space encoded as No. 4. Behavior settings for holidays or weekendssuch as school, office, park, and market-are space encoded as No. 5. Space levels encoded 1 to 4 are the main subjects of this study. Spaces outside the house (No. 5) are not considered for information conversion. Definitions for each level are shown in Table 1.

Table 1. Interior Space Encoding for the House

\begin{tabular}{lcl}
\hline $\begin{array}{l}\text { Sphere } \\
\text { Characteristics }\end{array}$ & $\begin{array}{l}\text { Sphere } \\
\text { Encoding }\end{array}$ & Name of Space \\
\hline & 1 & Bathroom, bedrooms \\
\cline { 2 - 3 } $\begin{array}{l}\text { The larger } \\
\text { the number, } \\
\text { the lower } \\
\text { the privacy }\end{array}$ & 2 & Kitchen, study room \\
\cline { 2 - 3 } & $\begin{array}{c}5 \text { (not } \\
\text { included) }\end{array}$ & Space outside the house \\
\hline
\end{tabular}

\subsection{Journal Encoding}

People's daily lives are filled with people, events, times, things, and places. This study begins with a space user's daily life sphere. Different from ordinary questionnaires and interviews, a space user records his or her daily activities and uses a journal to complete the recording of his or her daily behaviors. User journals allow the researcher to receive details of a space user's daily life. The descriptions of a space user's preferences, activities, environments, and uses of objects are therefore received through this mechanism. The interior designer thus obtains a complete script of life. The journal is recorded every hour, 24 hours a day, for 14 days. During the recording period, family members are required to record behaviors that occur in daily activities, along with where and when they happen. Samples from the journal encodings are given in Tables 2. through 4.

\subsection{Changes in the Life Sphere in a Day}

This section analyzes journal codes collected from the daily records in the case study. Visualization analysis is performed for changes in the life sphere during a day and for numeric data about the members' sphere distribution records during the test period. 
Table 2. Journal Encoding: A Day in Mr. Peng's Life

\begin{tabular}{|c|c|c|c|}
\hline Time & Activity & Name of Space & Sphere Encoding \\
\hline 12:00 AM & Sleeping & Bedroom & 1 \\
\hline $1: 00$ & Sleeping & Bedroom & 1 \\
\hline $2: 00$ & Sleeping & Bedroom & 1 \\
\hline 3:00 & Sleeping & Bedroom & 1 \\
\hline 4:00 & Sleeping & Bedroom & 1 \\
\hline 5:00 & Sleeping & Bedroom & 1 \\
\hline $6: 00$ & Sleeping & Bedroom & 1 \\
\hline $7: 00$ & Get up and wash & Bathroom & 1 \\
\hline $8: 00$ & Prepare breakfast & Kitchen & 2 \\
\hline 9:00 & Eat breakfast & Dining room & 3 \\
\hline 10:00 & Visit customers & $\begin{array}{l}\text { Space outside } \\
\text { the house }\end{array}$ & 5 \\
\hline 11:00 & Visit customers & $\begin{array}{l}\text { Space outside } \\
\text { the house }\end{array}$ & 5 \\
\hline 12:00 PM & Visit customers & $\begin{array}{l}\text { Space outside } \\
\text { the house }\end{array}$ & 5 \\
\hline 1:00 & Eat lunch & $\begin{array}{l}\text { Space outside } \\
\text { the house }\end{array}$ & 5 \\
\hline $2: 00$ & Go to work & $\begin{array}{l}\text { Space outside } \\
\text { the house }\end{array}$ & 5 \\
\hline 3:00 & Go to work & $\begin{array}{l}\text { Space outside } \\
\text { the house }\end{array}$ & 5 \\
\hline 4:00 & Go to work & $\begin{array}{l}\text { Space outside } \\
\text { the house }\end{array}$ & 5 \\
\hline $5: 00$ & Go to work & $\begin{array}{l}\text { Space outside } \\
\text { the house }\end{array}$ & 5 \\
\hline $6: 00$ & Prepare dinner & Kitchen & 2 \\
\hline $7: 00$ & Eat dinner & Dining room & 3 \\
\hline $8: 00$ & Eat dinner & Dining room & 3 \\
\hline 9:00 & Prepare project report & Study room & 2 \\
\hline 10:00 & Prepare project report & Study room & 2 \\
\hline 11:00 & $\begin{array}{l}\text { Take a bath and get } \\
\text { ready for bed }\end{array}$ & Bathroom & 1 \\
\hline
\end{tabular}

Table 3. Journal Encoding: A Day in Mrs. Peng's Life

\begin{tabular}{clll}
\hline Time & Activity & Name of Space & Sphere Encoding \\
\hline 12:00 AM & Sleeping & Bedroom & 1 \\
\hline $1: 00$ & Sleeping & Bedroom & 1 \\
\hline $2: 00$ & Sleeping & Bedroom & 1 \\
\hline $3: 00$ & Sleeping & Bedroom & 1 \\
\hline $4: 00$ & Sleeping & Bedroom & 1 \\
\hline $5: 00$ & Sleeping & Bedroom & 1 \\
\hline 6:00 & Sleeping & Bedroom & 1 \\
\hline $7: 00$ & Get up and wash & Bathroom & 1 \\
\hline 8:00 & Prepare breakfast & Kitchen & 2 \\
\hline $9: 00$ & Eat breakfast & Dining room & 3 \\
\hline $10: 00$ & Chore & Kitchen & 2 \\
\hline $11: 00$ & Chore & Dining room & 3 \\
\hline $12: 00$ PM & Prepare lunch & Kitchen & 2 \\
\hline 1:00 & Eat lunch & Dining room & 3 \\
\hline 2:00 & Nap time & Bedroom & 1 \\
\hline $3: 00$ & Nap time & Bedroom & 1 \\
\hline 4:00 & Walk the dog in the park & $\begin{array}{l}\text { Space outside } \\
\text { the house }\end{array}$ & 5 \\
\hline 5:00 & Go grocery shopping & $\begin{array}{l}\text { Space outside } \\
\text { the house }\end{array}$ & 5 \\
\hline 6:00 & Prepare dinner & Kitchen & 2 \\
\hline 7:00 & Eat dinner & Dining room & 3 \\
\hline 8:00 & Eat dinner & Dining room & 3 \\
\hline $9: 00$ & Do laundry & Balcony & 4 \\
\hline 10:00 & $\begin{array}{l}\text { Take a bath and get } \\
\text { ready for bed }\end{array}$ & Bathroom & 1 \\
\hline $11: 00$ & Sleeping & Bedroom & 1 \\
\hline & & & \\
\hline
\end{tabular}

Table 4. Journal Encoding: A Day in the Pengs's Son's Life

\begin{tabular}{|c|c|c|c|}
\hline Time & Activity & Name of Space & Sphere Encoding \\
\hline 12:00 AM & Sleeping & Bedroom & 1 \\
\hline $1: 00$ & Sleeping & Bedroom & 1 \\
\hline $2: 00$ & Sleeping & Bedroom & 1 \\
\hline $3: 00$ & Sleeping & Bedroom & 1 \\
\hline 4:00 & Sleeping & Bedroom & 1 \\
\hline 5:00 & Sleeping & Bedroom & 1 \\
\hline $6: 00$ & Sleeping & Bedroom & 1 \\
\hline $7: 00$ & Get up and wash & Bathroom & 1 \\
\hline $8: 00$ & Eat breakfast & Dining room & 3 \\
\hline 9:00 & Catch a bus & $\begin{array}{l}\text { Space outside } \\
\text { the house }\end{array}$ & 5 \\
\hline 10:00 & Go to school & $\begin{array}{l}\text { Space outside } \\
\text { the house }\end{array}$ & 5 \\
\hline $11: 00$ & Go to school & $\begin{array}{l}\text { Space outside } \\
\text { the house }\end{array}$ & 5 \\
\hline 12:00 PM & Go to school & $\begin{array}{l}\text { Space outside } \\
\text { the house }\end{array}$ & 5 \\
\hline $1: 00$ & Go to school & $\begin{array}{l}\text { Space outside } \\
\text { the house }\end{array}$ & 5 \\
\hline $2: 00$ & Go to school & $\begin{array}{l}\text { Space outside } \\
\text { the house }\end{array}$ & 5 \\
\hline $3: 00$ & Go to school & $\begin{array}{l}\text { Space outside } \\
\text { the house }\end{array}$ & 5 \\
\hline 4:00 & Go to school & $\begin{array}{l}\text { Space outside } \\
\text { the house }\end{array}$ & 5 \\
\hline $5: 00$ & Go to school & $\begin{array}{l}\text { Space outside } \\
\text { the house }\end{array}$ & 5 \\
\hline $6: 00$ & Catch a bus & $\begin{array}{l}\text { Space outside } \\
\text { the house }\end{array}$ & 5 \\
\hline $7: 00$ & Eat dinner & Dining room & 3 \\
\hline $8: 00$ & Eat dinner & Dining room & 3 \\
\hline 9:00 & Do homework & Study room & 2 \\
\hline 10:00 & Do homework & Study room & 2 \\
\hline $11: 00$ & $\begin{array}{l}\text { Take a bath and get } \\
\text { ready for bed }\end{array}$ & Bathroom & 1 \\
\hline
\end{tabular}

Family members' activities in the environment are presented by the hour; these are the maps of sphere changes in a day during the test period, as shown in Fig.2. (based on Tables 2., 3., and 4.). The measurement scale for life sphere change in a day is defined according to three principles:

(1) The line coordinates of the concentric circles have 24 gradations with each representing one hour. The number increases clockwise. One circle represents one day.

(2) The concentric circles are indicative of degrees of privacy. The farther the circle is from the center, the more open the sphere characteristics of the space it represents and the bigger the number.

(3) The colored area indicates that some family members were using that space at that particular time.

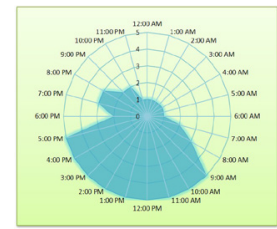

a) Mr. Peng

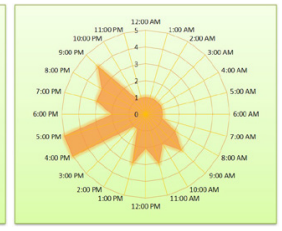

b) Mrs. Peng

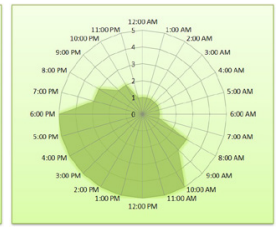

c) Pengs's Son
Fig.2. Maps of Sphere Change in Each Space in a Day 


\section{Discussion and Analysis}

4.1 Sphere Activity Change Index during the Test Period

Continuing from the members' maps of life sphere changes in the previous section, a two-week numerical analysis of the Pengs's sphere activity changes was performed. The results are shown in Tables 5. to 7. Two assessment indexes were derived: duration-days (D.D.) as in (1) and frequency-days (F.D.) as in (2). These two indexes show the sphere changes of the family members, the space sphere where each spent most of his or her time, and the frequency of visits to the bathroom, bedroom, kitchen, study room, dining room, living room, and balcony. In formula (1), this index estimates the time the family members spent in the space per day. In formula (2), this index estimates the number of visits to the space per day. The formulas of indexes D.D. and F.D. are given below:

$$
\text { D.D. }=\sum \frac{\text { Total time used for the space during the period of recording }}{\text { Days of recording }}
$$

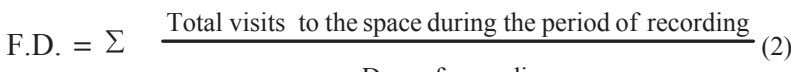

$$
\text { Days of recording }
$$

Table 5. Numerical Analysis for Mr. Peng's Sphere Changes During a Two-Week (14-day) Period

\begin{tabular}{llccl}
\hline Name of Space & $\begin{array}{l}\text { Total Time of } \\
\text { Usage (hours) }\end{array}$ & D.D. & Total Visits & F.D. \\
\hline Bathroom & 14.5 & 1.04 & 29 & 2.07 \\
\hline Bedroom & 97.0 & 6.93 & 19 & 1.36 \\
\hline Kitchen & 22.0 & 1.57 & 20 & 1.43 \\
\hline Study room & 16.5 & 1.18 & 8 & 0.57 \\
\hline Dining room & 19.5 & 1.39 & 23 & 1.64 \\
\hline Living room & 9.5 & 0.68 & 7 & 0.50 \\
\hline Balcony & 1.5 & 0.11 & 3 & 0.21 \\
\hline
\end{tabular}

Table 6. Numerical Analysis for Mrs. Peng's Sphere Changes During a Two-Week (14-day) Period

\begin{tabular}{lllll}
\hline Name of Space & $\begin{array}{l}\text { Total Time of } \\
\text { Usage (hours) }\end{array}$ & D.D. & Total Visits & F.D. \\
\hline Bathroom & 24 & 1.71 & 42 & 3 \\
\hline Bedroom & 121.5 & 8.68 & 47 & 3.36 \\
\hline Kitchen & 33 & 2.36 & 35 & 2.5 \\
\hline Study room & 10 & 0.71 & 7 & 0.5 \\
\hline Dining room & 34.5 & 2.46 & 34 & 2.43 \\
\hline Living room & 11.5 & 0.82 & 12 & 0.86 \\
\hline Balcony & 6 & 0.43 & 11 & 0.79 \\
\hline
\end{tabular}

Table 7. Numerical Analysis for Pengs's Son's Sphere Changes During a Two-Week (14-day) Period

\begin{tabular}{llccl}
\hline Name of Space & $\begin{array}{l}\text { Total Time of } \\
\text { Usage (hours) }\end{array}$ & D.D. & Total Visits & F.D. \\
\hline Bathroom & 13 & 0.93 & 32 & 2.29 \\
\hline Bedroom & 114.5 & 8.18 & 41 & 2.93 \\
\hline Kitchen & 5.5 & 0.39 & 11 & 0.79 \\
\hline Study room & 11 & 0.79 & 7 & 0.5 \\
\hline Dining room & 19 & 1.36 & 23 & 1.64 \\
\hline Living room & 6 & 0.43 & 5 & 0.36 \\
\hline Balcony & 2 & 0.14 & 4 & 0.29 \\
\hline
\end{tabular}

4.2 Visualization of Sphere Activity during the Period: Life Model Information Tree

A good visualization design not only has aesthetic value but also facilitates easier reading for the analysis and comparison of charts. In this section, the distribution of the family members' average time spent in each space sphere was visually simulated and then converted to graphic information according to sphere activity change indexes D.D. and F.D. Just as plants change under different conditions of light, water, and soil, the family members' "Life Model Information Tree," developed through this process, behaves similarly. The structure of the growth model is detailed below:

(1) Tree trunk: This is the center as well as the beginning of growth.

(2) Branches: This refers to the direction of growth. The number of spaces varies from one interior design project to another. The change and calculation of angles have a relationship with the number of spaces, thus generating different patterns.

(3) Segments of branches: The F.D. index is indicated by the length of a segment of the branch. The length of a segment represents the total visits to a space. The longer the segment, the more frequent the visitations to the space.

(4) Number of petals: The D.D. index is indicated by the number of petals, which represents the average amount of the time the family members spent in the space. The more petals there are, the more time spent in the space.

(5) Rule of growth: The growth model is divided into two basic elements - core and direction. A polygon represents the originating core of a tree trunk, while the text inside the polygon represents the appellation of the family members. The number of sides the polygon $(\mathrm{N})$ has depends on the number of spaces in the interior design project (Table 8.). The rule of growth for a single member's Life Model Information Tree is defined as follows: Multiple branch axes are generated and extended from the center, with the direction of each axis representing a space. The angle of each branch $(\theta)$ is obtained by evenly dividing 360 degrees by the number of spaces in the interior design project. For instance, as indicated in Fig.3., $\theta=360$ degrees $/ 6=60$ degrees. The length of a branch axis (F.D.) is derived from the frequency of a member's visits to each space, while the number of petals at the end of the branch axis is derived from the average amount of time spent in the space (D.D.). The Pengs's Life Model Information Tree is shown in Fig.4. (based on Tables 5., 6., and 7.).

Table 8. Growth Pattern of Life Model Information Tree

Triangle Square Pentagon Hexagon Heptagon Octagon




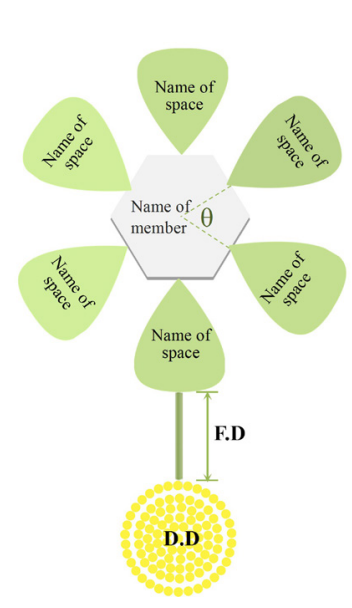

This is the no. of petals (D.D.):

This indicates the time the family members spent in a space per day. The area of the circle is scaled as $100 \%$. The area occupied by the petals indicates the percentage. The more petals there are, the more time spent in the space.

Length of branch segment (F.D.):

This indicates the total visits to a space. The total length of the segment is scaled as $100 \%$. The whole length is then divided into sections in proportion to the percentage of each part. The length of each segment indicates the percentage of each part. The longer the segment, the more frequent the visits to the space.

Fig.3. The Structure of the Life Model Information Tree

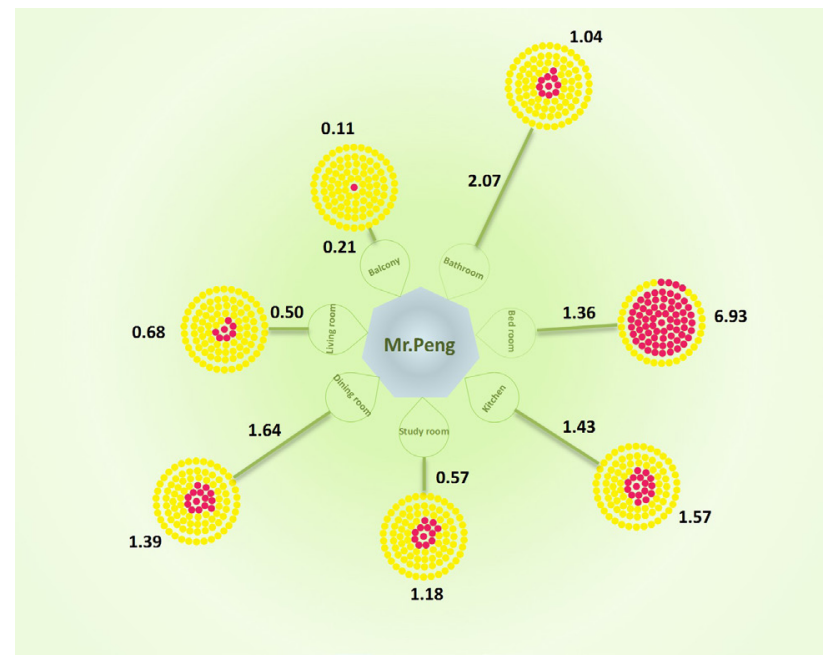

a) Mr. Peng

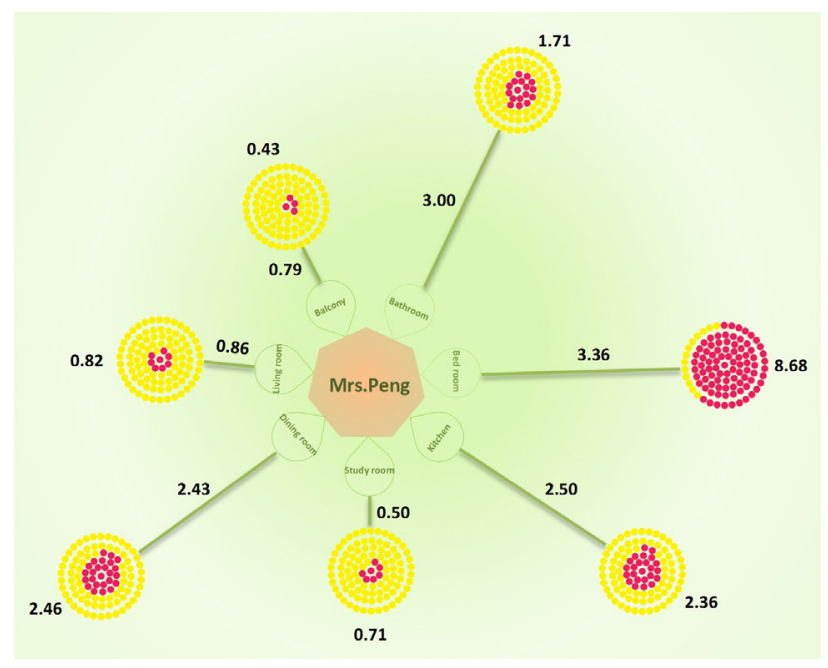

b) Mrs. Peng

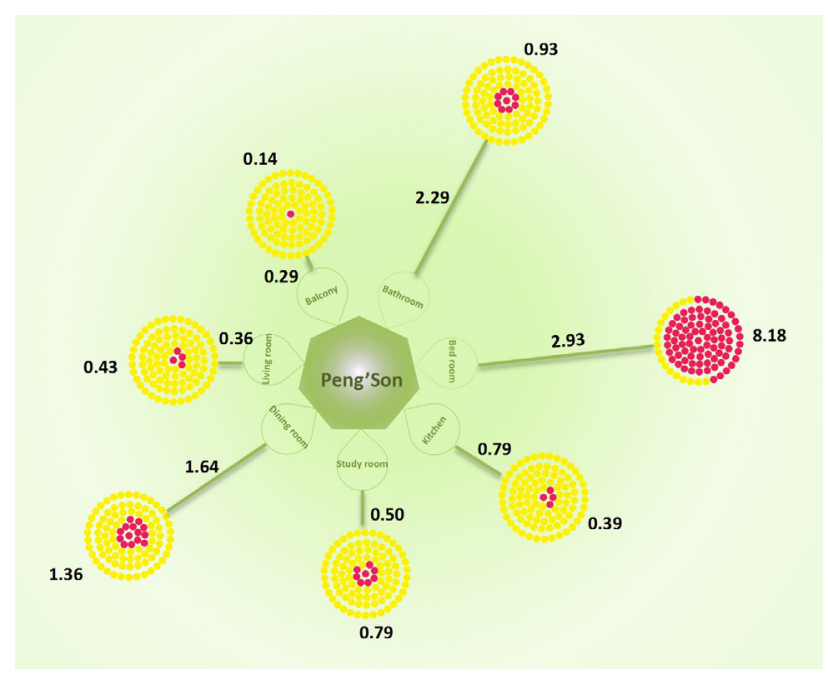

c) Pengs's Son

Fig.4. Representation of the Pengs's Life Model Information Tree

\section{Conclusion and Future Research}

This research created a method for providing an interior design data source that suited the objectives of this study. This paper also detailed how the consistency of the numerical data was maintained in the process of formatting. It will take a long time for project interior designers to find a periodic mode that is inherent in the data if only a simple electronic data table is provided. Although the current design outcome of the visualization is a static image, the whole iconic infrastructure and hierarchies have been established, which is the most difficult part of the task. Generally speaking, mass information may be converted into a meaningful image framework through information visualization. Compared to the pure digital displays used in the past, information visualization is easier and more intuitive in helping interior designers quickly understand and use information.

The life sphere change map defined and developed in this study can help interior designers understand family members' changes in the space sphere, from which their life models may be determined. Preferences and habits in space usage can be determined in accordance with combination of these types. The indexes D.D. and F.D., created by changes in sphere activity, can further highlight the space sphere an individual family member emphasizes and visualize the distribution of average time spent in each space sphere per day.

Follow-up studies should build on the current results and develop an algorithm for building an interactive information system platform with the visualized model of the Life Model Information Tree as its foundation. Different images may be created based on visits to the space and may vary with time. Images presented this way can sharply display the characteristics of the data and the differences among them, thus assisting interior designers in easily interpreting information related to the design. 
Moreover, interior designers may extract information more efficiently from the space users' journal records and in the model of the exported visualized results. More interior design service case studies may be recorded for further analysis of the space users' favorite data. The results may be stored in the experience base of the case study for future reference when making decisions on follow-up design elements. They may also be used as a basis for improvement and to determine whether the best result-one that fits the user's customization purpose - is produced.

\section{Acknowledgments}

This research was supported by the Ministry of Science and Technology of Taiwan (MOST-1032221-E-468 -014).

\section{References}

1) Barker, R. G. (1968) Ecological psychology: Concepts and method for studying the environment of human behavior. Stanford: Stanford University Press.

2) Bederson, B. B. and Shneiderman, B. (2003) The craft of information visualization: Readings and reflections. San Francisco: Morgan Kaufmann.

3) Card, S. K., Mackinlay, J. D., and Shneiderman, B. (1999) Readings in information visualization: Using vision to think. San Francisco: Morgan Kaufmann.

4) Cawthon, N. and Vande Moere, A. (2007) The effect of aesthetic on the usability of data visualization. IEEE International Conference on Information Visualization (IV'07), IEEE, Zurich, Switzerland, pp.637-648.

5) Colin, W. (2004) Information visualization: Perception for design. 2nd ed. San Francisco: Morgan Kaufmann.

6) Hillier, B. and Hanson, J. (1984) The social logic of space. Cambridge, UK: Cambridge University Press.

7) Hillier, B. (1996) Space is the machine: A configurational theory of architecture. Cambridge, UK: Cambridge University Press.

8) Højrup, T. (2003) State, culture, and life modes: The foundations of life mode analysis. London: Ashgate.

9) Holmes, T. G. (2007) Eco-visualization: Combining art and technology to reduce energy consumption. Proceedings of the 6th ACM SIGCHI Conference on Creativity and Cognition, New York, pp.153-162.

10) Huang, W., Matsushita, D., and Munemoto, J. (2006) Interactive evolutionary computation (IEC) method of interior work (IW) design for use by non-design-professional Chinese residents. Journal of Asian Architecture and Building Engineering, 5 (1), pp.91-98.

11) Huang, W. and $\mathrm{Xu}$, W. (2009) Interior color preference investigation using interactive genetic algorithm. Journal of Asian Architecture and Building Engineering, 8 (2), pp.439-445.

12) Lamping, J., Rao, R., and Pirolli, P. (1995) A focus +context technique based on hyperbolic geometry for visualizing large hierarchies. Proceedings of the CHI'95, ACM Conference on Human Factors in Computing Systems, New York, pp.401-408.

13) Lang, J. (1987) The behavior setting: A unit for environmental analysis and design. In Creating architectural theory: The role of the behavioral sciences in environmental design. New York: Van Nostrand Reinhold, pp.113-125.

14) Lewin, K. (1943) Defining the field at a given time. Psychological Review, 50, 292-310. Republished in Resolving social conflicts and field theory in social science. Washington, DC: American Psychological Association, 1997.

15) Schoggen, P. (1989) Behavior settings: A revision and extension of Roger G. Barker's ecological psychology. Stanford: Stanford University Press.
16) Shneiderman, B. (1992) Tree visualization with treemaps: A 2-D space-filling approach. ACM Transactions on Graphics, 11 (1), pp.92-99.

17) Skog, T., Ljungblad, S., and Holmquist, L. E. (2003) Between aesthetics and utility: Designing ambient information visualizations. INFOVIS 2003, pp.233-240.

18) Spence, R. (2001) Information visualization. Reading: AddisonWesley.

19) Stasko, J., Catrambone, R., Guzdial, M., and Mcdonald, K. (2000) An evaluation of space-filling information visualizations for depicting hierarchical structures. International Journal of HumanComputer Studies, 53 (5), pp.663-694.

20) Vande Moere, A. and Purchase, H. (2011) On the role of design in information visualization. Information Visualization Journal, 10 (4), pp.356-371.

21) Vitruvius, P. (1960) The ten books on architecture, trans. Morris Hicky Morgan. New York: Dover.

22) Wicker, A. W. (1987) Behavior setting reconsidered: Temporal stages, resources, internal dynamics, context. In D. Stokols and I. Altman (eds.), Handbook of environmental psychology. New York: John Wiley \& Sons, pp.613-653.

23) Wicker, A. W. (1992) Making sense of environments. In W. B. Walsh, K. H. Clark, and R. H. Price (eds.), Person-environment psychology: Models and perspectives. Hillsdale, NJ: Lawrence Erlbaum Associates, pp.158-191. 